\title{
The impact of the hormonal milieu and stress-associated hypothalamic dopaminergic activation on the rapid compensatory adrenal growth in cycling female rats
}

\author{
Anastasia Sfikakis, Pothitos M. Pitychoutis, Katerina Antoniou, Nikolaos Bikas, \\ Zeta Papadopoulou-Daifoti
}

Department of Experimental Pharmacology, Athens University Medical School, Athens, Greece

\begin{abstract}
OBJECTIVE: We sought to investigate the impact of emotional and surgical stress applied in two hormonally different estrous-cycle phases as well as the hypothalamic dopaminergic involvement on the rapid compensatory adrenal growth (CAG) and the individual adrenal growth (IAG) in rats. DESIGN: After surgery for left and sham adrenalectomy (Adx) carried out on either diestrus-2 (DE-2) or proestrus (PE), CAG and IAG were evaluated in PE or estrus (E), respectively. Hypothalamic dopaminergic activity was also assessed by measuring dopamine (DA) and 3,4-dihydroxyphenylacetic acid (DOPAC) levels in DE-2, PE and E in controls and in $P E$ and $E$ in sham and left adrenalectomized rats. All groups experienced similar chronic emotional stress assessed by the emotional reactivity score recorded during vaginal screening. RESULTS: In controls, DOPAC levels in DE-2 were higher than in PE and E. In PE, following surgery in DE-2, DOPAC levels and DOPAC/DA ratio were increased compared to PE controls; CAG was not significant, while negative correlations of IAG and CAG with DOPAC/DA ratio were observed. In E, CAG and IAG were significant, while dopaminergic activity was not increased compared to $E$ controls. CONCLUSIONS: The significant CAG and IAG following left Adx in PE but not in DE-2 suggest a pronounced positive impact of the hormonal milieu on this process. The profound attenuation of CAG and IAG in PE suggests a negative effect of stress-associated dopaminergic activation.
\end{abstract}

Key words: Compensatory and individual adrenal growth, Diestrus-2, Hypothalamic dopamine, Proestrus, Stress

Address for correspondence:

Anastasia Sfikakis, Department of Experimental

Pharmacology, Medical School, University of Athens,

75 Mikras Asias Str., GR-11527, Athens, Greece,

Tel: +30210 8040964, Fax: +30210 7462554,

e-mail: ansfikaki@med.uoa.gr

Received 21-04-08, Revised 20-07-08, Accepted 30-08-08

\section{INTRODUCTION}

Compensatory adrenal growth (CAG) after unilateral adrenalectomy (Adx) is a well established model of adrenal growth in rats. ${ }^{1}$ After left Adx, the increase in wet weight, protein, DNA and RNA content of the remaining right adrenal within $12-24 \mathrm{~h}$, 
known as the early rapid CAG, has been attributed to a neurally mediated reflex between the adrenal and the hypothalamus. ${ }^{2,3}$ Despite the homeostatic significance of CAG, there are no studies to illustrate the early rapid CAG following unilateral adrenalectomy in cycling female rats carried out in two consecutive but hormonally different phases of the 4-day estrous cycle. This 4-day cycle includes 4 phases, defined by vaginal smears: 1) Diestrus-2 (DE-2), 2) Proestrus (PE), 3) Estrus (E) and 4) Diestrus-1 (DE-1), also referred to as metestrus. ${ }^{4-7}$ Hormonal determinations (estradiol, progesterone, and $\mathrm{LH}$ ) throughout these 4 phases $^{5}$ has shown profound differences between the two consecutive phases DE-2 and PE, the peak levels of these three hormones being found in PE. Details of the hormonal milieu of the various phases of the 4-day estrous cycle in the rat can be found in the study of Butcher et al. ${ }^{5}$

Considering that a study in cycling rats requires vaginal smear screening (VSS), we should mention that in one of our studies in cycling rats we found that the procedure of VSS represented a mild stress shown by a rise in ACTH about 10 min following VSS. Furthermore, by recording behavioural indices during this procedure we found that the final emotional reactivity score (ERS), which expresses fear and resistance to VSS, was higher after prolonged (12-18 days) compared to short duration (7-10 days) VSS. The prolonged VSS was associated with higher adrenal weight, reduced thymus weight and reduced ACTH response to 2.5 min ether stress. ${ }^{8}$ In a subsequent study, an estrous-cycle phase effect was noted on the hypothalamic dopaminergic status, expressed by dopaminergic alterations in DE-2 but not on the phases $\mathrm{PE}$ and E. ${ }^{9}$

In the present study, we sought to investigate the impact of chronic emotional stress and surgical stress applied in two profoundly different phases of the estrous cycle (DE-2 and PE), on the rapid CAG and individual adrenal growth (IAG) and in relation to the neurochemical dopaminergic hypothalamic status. It must be mentioned that the day of DE-2, which precedes the ovulatory surge taking place in $\mathrm{PE}$, might correspond to the follicular phase of the human cycle, while PE might correspond to the periovulatory phase. CAG is defined as the growth seen in the remaining adrenal of a unilaterally adrenalectomized
(Adx) rat relative to the weight of the corresponding adrenal of a rat with both adrenals, which underwent only sham operation under the same conditions. ${ }^{1-3}$ IAG is defined as the growth seen in the remaining adrenal relative to the corresponding removed adrenal in the same rat.

The IAG in humans following unilateral adrenalectomy can be estimated by comparison of the size of the remaining adrenal (estimated by magnetic resonance imaging) with the size of the removed adrenal. Because of the need for surgical intervention in a number of patients with adrenal mass ${ }^{10,11}$ and the high prevalence of unilateral incidentalomas (90\% versus $10 \%$ bilateral), ${ }^{12,13}$ a research study in rats, which includes CAG and IAG assessment in relation to estrous cycle, stress and hypothalamic dopaminergic status, besides its theoretical interest, might have implications in humans with nosology requiring unilateral adrenalectomy.

\section{MATERIALS AND METHODS}

\section{Animals and general procedure}

Virgin female rats of the Wistar strain, born and raised in our laboratory under controlled conditions (14 h light-10 h dark, lights on from 06:00 to 20:00 h, $21-23^{\circ} \mathrm{C}$ ), were studied at 3-4 months of age. Rat chow and water were provided ad libitum. Only rats characterized by at least 3 consecutive 4-day estrous cycles were included in this study. The number of studied rats $(n=58)$ included 24 control rats and 34 rats that were exposed to surgery: 16 for sham and 18 for left Adx carried out on the day before autopsy. In all rats dopamine (DA) and its metabolite 3,4-dihydrophenylacetic acid (DOPAC) were measured in the hypothalamus, including the preoptic area.

\section{Procedure for Vaginal Smear Screening (VSS)}

The handling for obtaining vaginal smears consisted of grasping the rat gently but firmly by the skin of the neck and back to immobilize it in the upright position in order to insert with caution a cotton-swab humidified in drops of saline into the vagina and obtain vaginal cytology by hemicircular movement. The procedure for VSS has been proven to be stressful because the number of reactions recorded in the present study during handling for weighing, which 
preceded VSS for 4-5 days, was markedly lower than that recorded when VSS was added to handling for weighing in the same rats. The protocol used to achieve the chronic emotional stress related to VSS and the kind of different reactions recorded have been described in two previous studies. ${ }^{914}$ The sum of these reactions for each rat constituted the final emotional reactivity score (ERS), used as an index of the extent of experienced chronic emotional stress by each rat. The majority of rats had high ERS. In order to obtain groups with similar final ERS, rats with low ERS were distributed in the 7 groups examined for dopaminergic status in the hypothalamus with 2 rats in each group of 8 or 9 rats. At the end of the experiment the total number of days with handling in the 7 studied groups was similar, ranging from $16.22 \pm 1.29$ to $17.60 \pm 1.15$. The final ERS in PE and $E$ in left Adx rats were $15.10 \pm 3.08$ and $15.0 \pm 2.36$, respectively, while in sham Adx the corresponding values were $14.0 \pm 2.7$ and $15.0 \pm 1.86$. In the three groups of control rats the final ERS in DE-2, PE and E were 15.0 \pm 3.5 , $15.0 \pm 3.38$ and $15.7 \pm 3.98$, respectively.

\section{Assessment of the phases of the estrous cycle}

The phase of the estrous cycle was determined by VSS carried out between 09:00 and 11:00 h. The procedure for VSS is described in detail in previous studies. ${ }^{6-9,14}$ At autopsy, the phase of the estrous cycle was also assessed by taking the weight of the uterus.

\section{Surgical procedure}

Two groups of rats were subjected to surgery (one for sham and the other for left Adx) either in DE-2 or in PE. The surgical procedure for sham or left Adx was performed between 14:00 to 16:00 h, either in DE-2 or PE. The anesthetized rat was removed from a ventilated Plexiglas chamber filled with ether vapours after 2.5 min of exposure. During surgery, ether was administered again if needed. The surgical procedure lasted for about $15 \mathrm{~min}$ in all rats. In sham Adx, after incision through the skin and a cut through the muscle layer as in left Adx, the left adrenal was exposed but not touched. After the removal of the left adrenal and after sham Adx, retroperitoneal, muscle and skin incisions were closed by separate suturing. Littermates on the same cycle phase for sham and left Adx were used and equal timing for surgical procedures at sham and left Adx was kept in order to avoid an artefact due to unequal duration of surgery. ${ }^{15}$

\section{Procedures at autopsy}

All rats were sacrificed by decapitation between 09:00 and 11:00 h, 19-20 hours post surgery either in $\mathrm{PE}$ or E phases, immediately after assessment of emotional reactivity. In all studied rats including controls in DE-2, the brain was removed at autopsy and the hypothalamus was rapidly dissected on ice and weighed. In all rats the left and the corresponding right adrenal gland were weighed separately to the nearest $0.01 \mathrm{mg}$, after being trimmed of surrounding fat and connective tissue on a filter paper saturated with saline. In left Adx rats the weight of the removed left adrenal in each rat was compared to the remaining adrenal that was weighed at autopsy. All animal experiments were reviewed and approved by the local committee and were carried out in accordance with the European Communities Council Directive of 24 November 1986 (86/609/EEC).

\section{Measures of CAG and IAG}

Rapid (within 19-20 h) IAG was determined by using 3 indices: 1) the weight difference between the right and the corresponding, previously excised, left adrenal; 2) by direct comparison of the remaining right to the corresponding removed left adrenal; and 3) by the ratio of the remaining/corresponding removed adrenal weight, expressing the extent of IAG. CAG was estimated by comparison of the weight of the right adrenals between left Adx and sham Adx rats performed on the same day of the estrous cycle. A second method of analysis initiated in our laboratory was also applied..$^{15}$ This method compares the normalized right adrenal between sham and left Adx rats as previously described. ${ }^{16}$

\section{Neurochemical assessment}

After sacrifice by decapitation, the brain was removed and the hypothalamus was rapidly dissected on ice in all studied rats. The piece of this area included the whole hypothalamic tissue and the optic chiasm. The boundaries of the whole hypothalamic area were assessed according to Figure 92 of the Paxinos rat brain atlas. ${ }^{17}$ After immediate weighing, the tissue was homogenized and deproteinized in 
$500 \mu \mathrm{l}$ of $0.2 \mathrm{~N}$ perchloric acid solution containing $7.9 \mathrm{mM} \mathrm{Na}_{2} \mathrm{~S}_{2} \mathrm{O}_{5}$ and $1.3 \mathrm{mM} \mathrm{Na} \mathrm{N}_{2}$ EDTA. The homogenate was centrifuged at $14000 \mathrm{rpm}$ for $30 \mathrm{~min}$ and the supernatant was stored at $-80^{\circ} \mathrm{C}$. Analysis of DA and its metabolite DOPAC was performed by High-Performance Liquid Chromatography with an electrochemical detector (HPLC-ED).$^{18} \mathrm{~A}$ reversephase ion pair chromatography was used to assay DA and DOPAC in all samples. ${ }^{19}$ Details concerning the procedure have been previously described. ${ }^{9}$ The sensitivity of the assay was always tested by the use of external DA and DOPAC standards (Sigma, St. Louis, MO). The limit of detection was $1 \mathrm{pg} / 20 \mu \mathrm{l}$ (injection volume). Additionally, the DA turnover ratio DOPAC/DA ratio as indicative of the dopaminergic function was also calculated. Results are expressed as $\mu \mathrm{g} / \mathrm{g}$ of tissue.

\section{Statistics}

All values are presented as mean \pm SEM. One-way analysis of variance (ANOVA), followed by Scheff post hoc tests, was used to determine significant differences concerning neurochemical activity among groups. The significance of the difference between the remaining right and the corresponding removed left adrenal was determined by Student's paired t-test. Correlation statistical analysis (Pearson's correlation) was performed to investigate possible statistically significant correlations between IAG, normalized right adrenal, uterine weight, DOPAC levels and DOPAC/DA ratio. Statistical significance by Student's t-test between two independent groups was evaluated at the $2 \mathrm{P}$ level. Logarithmic conversion of values was performed when normality assumption was not valid. The level of statistical significance was set to $5 \%$.

\section{RESULTS}

The IAG value (Figure 1) was higher in E following left Adx in PE when compared to PE following left Adx in DE-2. Comparison by paired t-test of the remaining right adrenal weight to the corresponding removed left (Table 1) revealed a highly significant IAG in E while no IAG was detected in PE. The IAG in these rats when expressed by the ratio of remaining right adrenal weight in $\mathrm{E}$ to the corresponding removed left adrenal weight in PE was also higher than the ratio remaining right adrenal in $\mathrm{PE} /$ removed left in DE-2.

No significant CAG was observed in PE (Figure 2)

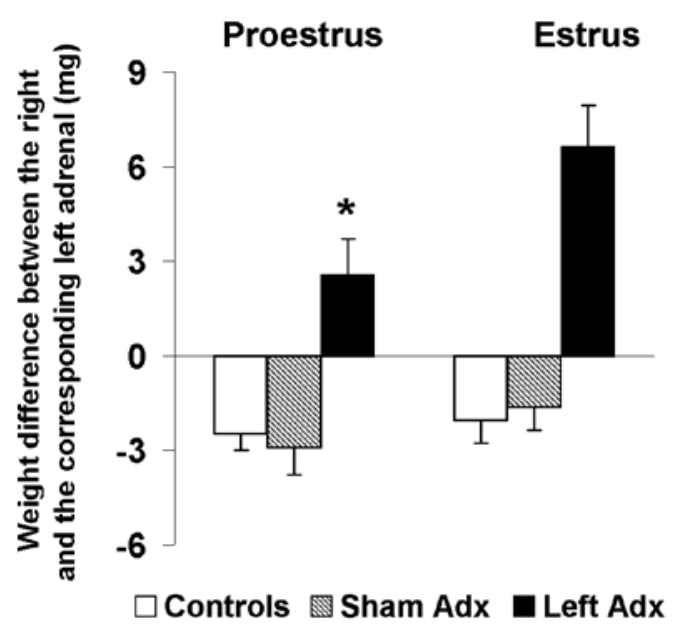

Figure 1. Weight differences in mg detected by subtracting the left adrenal from the corresponding right in 8 control, 8 sham Adx and IAG in 9 left Adx rats in proestrus and estrus. Data are presented as means \pm SEM $* \mathrm{P}<0.05$ for difference between proestrus and estrus in left Adx rats. Weight of the left adrenal is greater than the right in controls and sham operated, as expected. ${ }^{15,16}$

Table 1. Individual adrenal growth (IAG) in two consecutive estrous cycle phases, namely proestrus and estrus, assessed 19-20h post left Adx carried out in diestrus- 2 and proestrus, respectively.

\begin{tabular}{|c|c|c|c|}
\hline \multicolumn{2}{|c|}{ Proestrus IAG } & \multicolumn{2}{|c|}{ Estrus IAG } \\
\hline $\begin{array}{l}\text { Removed left adrenal } \\
\text { weight }(\mathrm{mg})\end{array}$ & $\begin{array}{c}\text { Remaining right adrenal } \\
\text { weight (mg) }\end{array}$ & $\begin{array}{c}\text { Removed left adrenal } \\
\text { weight (mg) }\end{array}$ & $\begin{array}{c}\text { Remaining right adrenal } \\
\text { weight }(\mathrm{mg})\end{array}$ \\
\hline (9) $25.18 \pm 1.75$ & (9) $27.77 \pm 2.30$ & (9) $28.18 \pm 1.67$ & (9) $34.83 \pm 1.89 * *$ \\
\hline \multicolumn{2}{|c|}{\begin{tabular}{|l} 
Proestrus \\
\end{tabular}} & \multicolumn{2}{|c|}{ Estrus } \\
\hline
\end{tabular}

(9) $1.12 \pm 0.03$

(9) $1.24 \pm 0.04 \dagger$

The values are expressed as means \pm S.E.M. Number of samples in parenthesis. $\uparrow \mathrm{P}<0.05$ difference between estrus and proestrus;

$* * \mathrm{P}<0.01$ differences between remaining right and corresponding removed left adrenal. 
when the remaining right adrenal of Adx rats is compared with the right adrenal of the corresponding sham Adx rats. The same results were obtained after comparing the normalized right adrenal. In contrast, significant CAG was observed in E. In left Adx rats

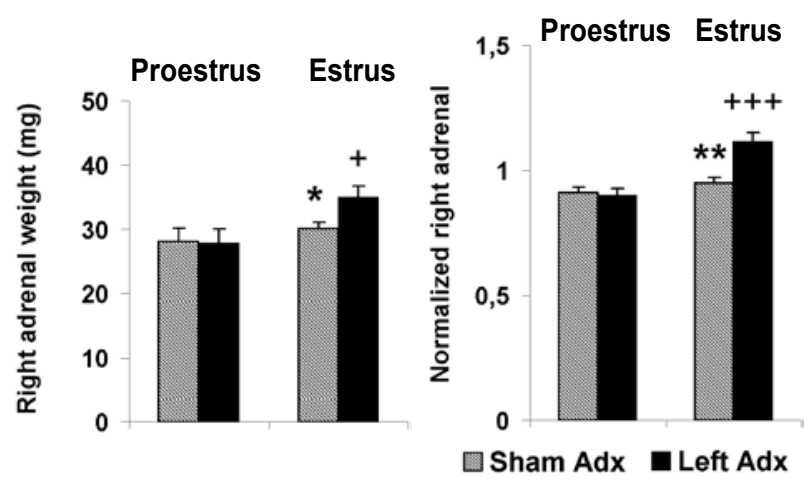

Figure 2. Comparison of rapid CAG between proestrus and estrus following left ADX carried out in diestrus- 2 and proestrus, respectively. ${ }^{*} \mathrm{P}<0.05,{ }^{*} \mathrm{P}<0.01$ for differences between sham and corresponding left Adx rats in estrus. Data are presented as means \pm SEM for 8 specimens in Sham Adx and 9 specimens in left Adx rats, respectively. ${ }^{+} \mathrm{P}<0.05,{ }^{+++} \mathrm{P}<0.001$ for differences between estrus and proestrus in left Adx rats. the weight of the right adrenal in E was significantly higher than the corresponding right adrenal weight in $\mathrm{PE}$, as was the normalized right adrenal in $\mathrm{E}$ versus PE. In sham Adx rats there was no difference in the right adrenal weight and the normalized right adrenal between PE and $\mathrm{E}$. This finding permitted the use of the normalized right adrenal in left Adx rats as an index of the extent of CAG. At autopsy, body weight in PE and E did not differ significantly between left and sham Adx rats.

Concerning hypothalamic dopaminergic status in control, sham and left Adx cycling rats, the following were detected (Figure 3). In the control group, DOPAC levels $(\mu \mathrm{g} / \mathrm{g})$ were significantly higher in DE-2 compared to PE and E. In sham Adx rats DOPAC levels in PE did not differ compared to DE-2 of controls. In left Adx rats DOPAC levels were not different in PE compared to DE-2 of controls, while DOPAC levels and DOPAC/DA ratio were higher compared to PE of controls. In this phase (PE) the IAG was non-significant (Table 1) and CAG was not apparent (Figure 2). In E in sham and in left Adx rats, DOPAC levels and DOPAC/DA ratio were not different from

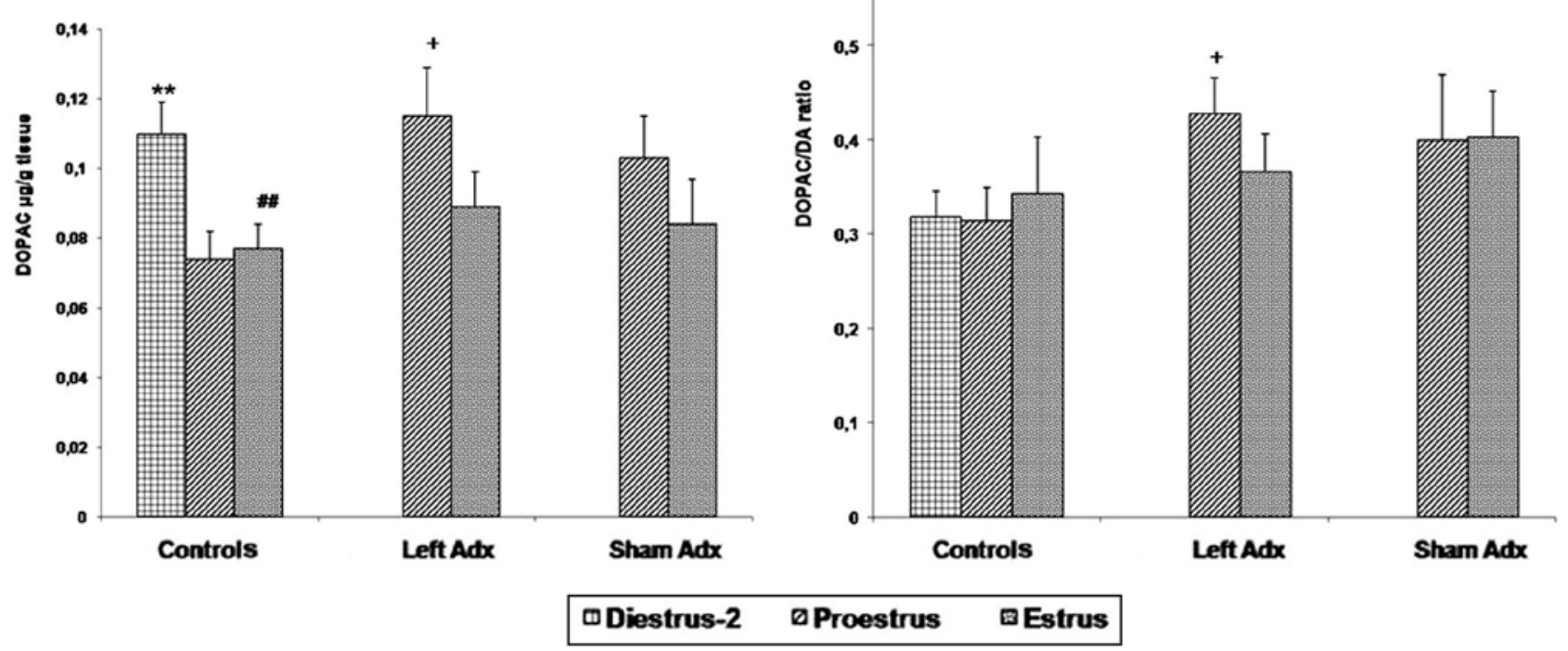

Figure 3. Hypothalamic dopaminergic status in diestrus-2 (DE-2), proestrus (PE) and estrus (E) in control rats and in PE and E in sham and left Adx rats. DA concentrations in 8 controls in DE-2: $0.348 \pm 0.058 \mu \mathrm{g} / \mathrm{g} ; 8$ in PE: $0.239 \pm 0.011 \mu \mathrm{g} / \mathrm{g} ; 8$ in E: $0.244 \pm 0.023$ $\mu \mathrm{g} / \mathrm{g}, 8$ sham Adx in PE: $0.284 \pm 0.027 \mu \mathrm{g} / \mathrm{g} ; 8$ in E: $0.221 \pm 0.021 \mu \mathrm{g} / \mathrm{g}$ and 9 left Adx rats in PE: $0.256 \pm 0.027 \mu \mathrm{g} / \mathrm{g} ; 9 \mathrm{in} \mathrm{E:} 0.250 \pm 0.017$ $\mu \mathrm{g} / \mathrm{g}$. Data are presented as means \pm SEM for 8 specimens in DE-2, PE and E in controls, for 8 specimens in PE and E in Sham Adx rats and for 9 specimens in $\mathrm{PE}$ and $\mathrm{E}$ in left $\mathrm{Adx}$ rats. ${ }^{*} \mathrm{P}<0.01$ compared to $\mathrm{PE}$ controls; ${ }^{\# \#} \mathrm{P}<0.01$ compared to DE- 2 controls; ${ }^{+} \mathrm{P}<0.05$ difference between left Adx and control rats in $\mathrm{PE}$. 
the levels found in controls, while the IAG and CAG were significant (Table 1 and Figure 2).

Regarding rats that underwent surgery for left and sham Adx, the 4-day estrous cyclicity seemed not to be affected as shown by vaginal smears and the weight of the uterus. In left $(\mathrm{N}=4)$ and sham $(\mathrm{N}=4)$ Adx rats, DOPAC levels measured at sacrifice in PE following surgery in DE-2 [ $\mathrm{N}=8$ rats; $0.093 \pm 0.0094 \mu \mathrm{g} / \mathrm{g}]$ were higher than those found at sacrifice in $\mathrm{E}$, following surgery in $\mathrm{PE}[\mathrm{N}=8$ rats; $0.068 \pm 0.0069 \mu \mathrm{g} / \mathrm{g}]$, the difference reaching significance $[\mathrm{t}=2.144, \mathrm{P}=0.05]$. No difference in final ERS in PE $(17 \pm 3.4)$ versus $E$ $(18 \pm 1.9)$ was detected. Although DOPAC/DA ratio was higher, the difference did not reach significance [0.357 \pm 0.035 in PE versus $0.318 \pm 0.048$ in E].

In Table 2 the dopaminergic status in left Adx rats in PE in relation to the final ERS is presented after separation into two groups using the mean value of the final ERS [15.11 \pm 3.08$]$. We arbitrarily formed 2 groups, one with ERS values above the overall mean $[24 ; 23 ; 30 ; 19]$ and another below the mean $[12 ; 10$; $10 ; 4 ; 4]$. In these two groups the IAG was also estimated. The results showed dopaminergic activation in rats with high ERS and an inverse relationship to IAG without difference in the days of handling.

Correlation analyses presented for all left Adx rats (Table 3 ) revealed that IAG was negatively correlated with DOPAC levels and DOPAC/DA ratio only in PE (Figure 4). Additionally, normalized right adrenal

Table 2. Chronic emotional stress-associated hypothalamic dopaminergic activation is inversely related to rapid individual adrenal growth.

\begin{tabular}{lcc}
\hline & $\begin{array}{c}\text { High chronic } \\
\text { emotional } \\
\text { stress }(\mathbf{n}=\mathbf{4})\end{array}$ & $\begin{array}{c}\text { Low chronic } \\
\text { emotional } \\
\text { stress }(\mathbf{n = 5})\end{array}$ \\
\hline Final emotional score & $24 \pm 2.27^{* * *}$ & $8 \pm 1.67$ \\
DA $(\mu \mathrm{g} / \mathrm{g})$ & $0.260 \pm 0.0135$ & $0.266 \pm 0.029$ \\
DOPAC $(\mu \mathrm{g} / \mathrm{g})$ & $0.134 \pm 0.008$ & $0.092 \pm 0.019$ \\
DOPAC/DA ratio & $0.523 \pm 0.025^{*}$ & $0.344 \pm 0.066$ \\
IAG remaining/removed & $1.015 \pm 0.042^{*}$ & $1.166 \pm 0.043$ \\
adrenal weight ratio & $0.125 \pm 1.09^{*}$ & $4.56 \pm 1.28$ \\
IAG $(\mathrm{mg})$ & $16.5 \pm 2.32$ & $15.0 \pm 2.01$ \\
Days with handling & & \\
***P<0.001; *P<0.05, High versus Low chronic emotional & & \\
stress & &
\end{tabular}

Table 3. Pearson's correlation coefficients for multiple comparisons of individual adrenal growth (IAG), normalized right adrenal, DOPAC levels, DOPAC/DA ratio and uterine weight in rats on proestrus and estrus respectively.

\begin{tabular}{lccc}
\hline & \multicolumn{3}{c}{ Proestrus } \\
\cline { 2 - 4 } & DOPAC & DOPAC/DA & Uterine weight \\
\hline IAG & $-0.698^{*}$ & $-0.704^{*}$ & 0.151 \\
Normalized & -0.664 & $-0.697^{*}$ & -0.012 \\
right adrenal & \multicolumn{3}{c}{ Estrus } \\
\hline \multicolumn{3}{c}{ DOPAC/DA } & Uterine weight \\
\cline { 2 - 4 } & DOPAC & DOPA & $0.708^{*}$ \\
IAG & 0.066 & 0.357 & 0.679 \\
Normalized & 0.057 & 0.382 & \\
right adrenal & & & \\
\hline
\end{tabular}

Statistically significant correlations; ${ }^{*} \mathrm{P}<0.05$;
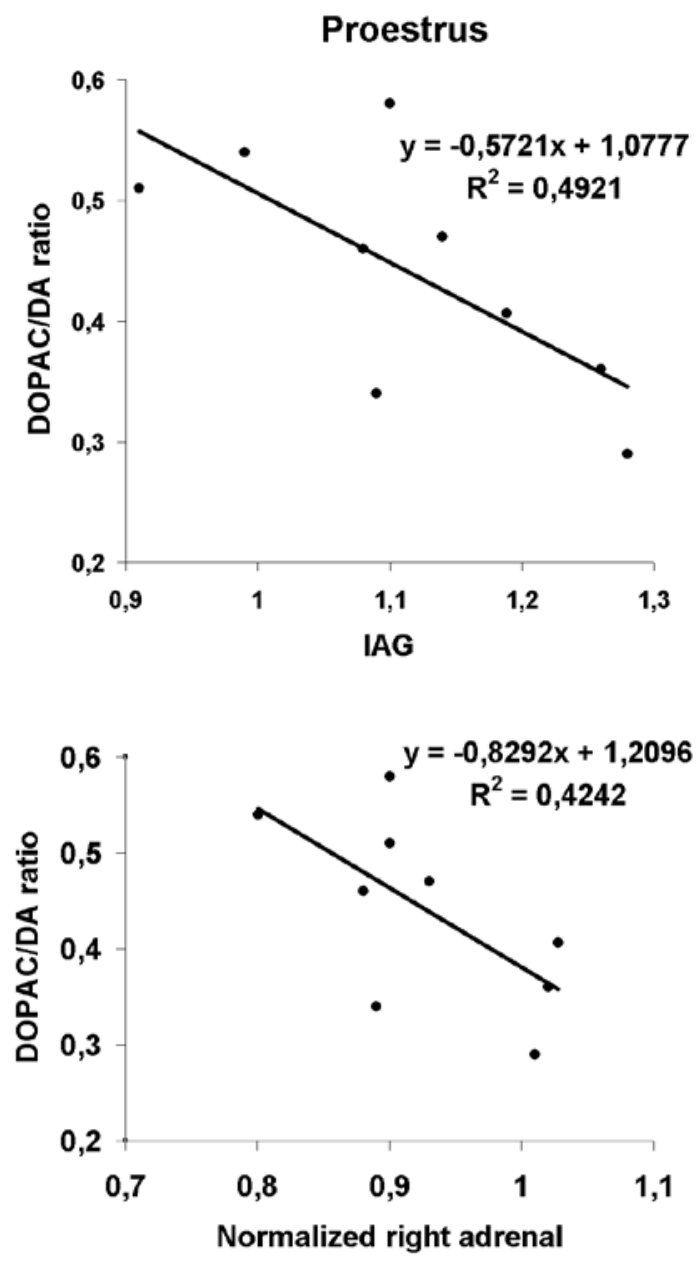

Figure 4. Negative correlation of IAG and normalized right adrenal in left Adx rats, with DOPAC/DA ratio in proestrus. Linear trendlines and corresponding line equations are also shown. 


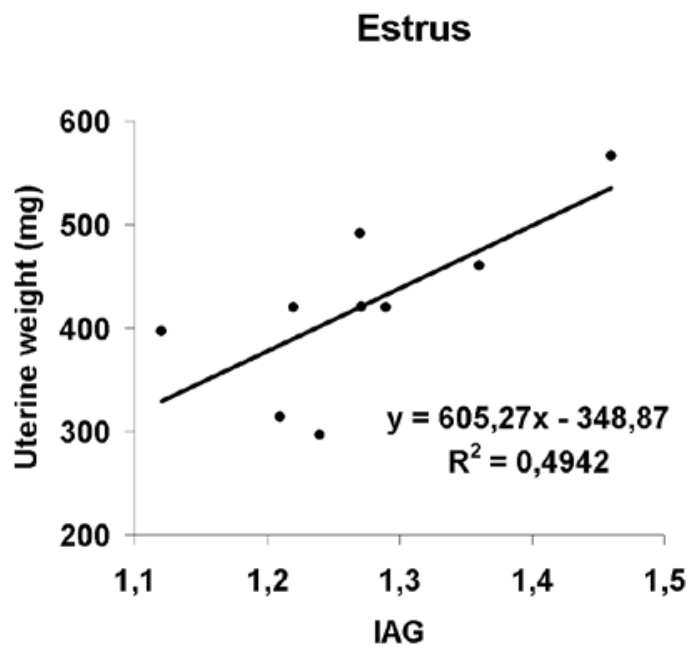

Figure 5. Positive correlation of IAG with uterine weight in left Adx rats in estrus. Linear trendlines and corresponding line equations are also shown.

in left Adx rats, showing the extent of CAG, was also negatively correlated with DOPAC/DA ratio. No statistically significant correlation of IAG with DOPAC and DOPAC/DA ratio was found in E. On the other hand, the uterine weight was positively correlated with IAG only for left Adx rats in E (Figure 5).

\section{DISCUSSION}

The results of the present study in 4-day cycling rats subjected to left Adx on either DE-2 or PE indicate that the CAG and IAG determined $19-20 \mathrm{~h}$ post surgery were significantly enhanced in rats examined in $\mathrm{E}$ but not in those examined in PE. The weight of the left adrenal was found higher compared to the right in control and sham Adx rats. This finding confirmed our previous unpublished results showing that in $87 \%$ of 64 adult cycling rats the left adrenal gland was heavier than the corresponding right. The remaining right adrenal 19-20 h after left Adx was found heavier than the corresponding removed left, showing a rapid IAG both in PE and $\mathrm{E}$ with the difference being higher in E compared to PE. On the other hand, the IAG was found significant only in $\mathrm{E}$ as was the CAG. The extent of $\mathrm{CAG},{ }^{16}$ by comparison of the normalized right adrenal between $\mathrm{PE}$ and $\mathrm{E}$ in left Adx rats, was also found higher in E. In our previous study, ${ }^{6}$ estradiol levels measured around the time Adx was performed in the present study (14:0016:00 h) were higher in PE compared to the other 3 phases of the estrous cycle; the two phases preceding PE (DE-1 and DE-2) and the phase following PE (E). The results on estradiol are in accordance with results found by Butcher et al ${ }^{5}$ throughout these 4 cycle phases. Uterine weight measured at autopsy at 0900 to $1100 \mathrm{~h}$ in the present study was found higher in PE compared to DE-2 in the control rats in agreement with our previous data. ${ }^{6,9}$

The aforementioned phase-related differentiation between DE-2 and PE with regard to both CAG and IAG response could be attributed in part to a delayed onset and prolonged duration "genomic" effect of estradiol. ${ }^{20}$ Notably, elevated corticotropin releasing hormone (CRH) mRNA in the afternoon of PE in the parvocellular paraventricular nuclei $(\mathrm{PVN})$ in the female rat has been reported. ${ }^{21}$ It has also been shown that human $\mathrm{CRH}$ gene expression is under direct estrogen regulation, ${ }^{22}$ raising the possibility of a molecular interplay between estradiol and $\mathrm{CRH}$ in the control of CAG and IAG. In support of this view, pro-opiomelanocortin (POMC)-derived ACTH levels were higher in the afternoon of $\mathrm{PE}$ in comparison to the other phases of the rat's estrous cycle. ${ }^{23}$ Thus, in the afternoon of $\mathrm{PE}$, under basal conditions an increase of $\mathrm{CRH}$-induced release of POMC from the anterior pituitary ${ }^{24}$ may be expected compared to DE-2. Accordingly, POMC processing in the anterior pituitary ${ }^{25}$ might result in higher amounts of the mitogenic precursor pro-opiomelanocortin derived $\mathrm{N}$-terminal peptide N-POMC 1-74 (pro- $\gamma$-MSH) in the circulation in PE compared to DE-2. The proteolytic cleavage of this precursor at the adrenal gland was proposed as the major mechanism of CAG control. ${ }^{26,27}$ In two later studies, ${ }^{28,29}$ an adrenal serine protease, cloned in rats, was found to be up-regulated following left Adx and capable of cleaving N-POMC (1-74) into the mitogenic fragment N-POMC (1-52) in the adrenal. Based on the aforementioned studies, the enhancement of CAG and IAG observed in E but not in $\mathrm{PE}$ could be mediated by an enhanced CRH-driven increase in the release of N-POMC (1-74) following surgery in $\mathrm{PE}^{21-23}$ but not in DE-2.5 The increase in N-POMC (1-74) could result in higher amounts of the mitogenic fragment in the adrenal.

DOPAC levels in PE and $\mathrm{E}$ were lower than in DE-2 in non-surgical controls. Following surgery in sham and left Adx rats, DOPAC levels in PE did 
not differ significantly compared to DE-2 controls. On the other hand, in left Adx rats but not in sham Adx, DOPAC levels and DOPAC/DA ratio were significantly higher in PE compared to PE controls. This difference between rats undergoing left adrenalectomy and sham Adx rats could be attributed to the extra manipulation caused by the removal of one adrenal to which sham Adx rats were not exposed. The overall results suggest that in left Adx rats the neural interference of chronic-stress associated hypothalamic dopaminergic activity in DE-2, being enhanced 19-20 $h$ post surgery in DE-2, contributed to the attenuation of IAG and to the absence of CAG in PE compared to $\mathrm{E}$. This assumption is supported by our results showing a significant negative correlation of the IAG and of the extent of CAG to the DOPAC/DA ratio in left Adx rats only in PE.

The negative effect of emotional stress-associated dopaminergic activation on IAG in PE is supported by the results showing that increased DOPAC/DA ratio in left Adx rats with very high ERS is associated with lower IAG, when compared to rats with low final ERS. The significant negative correlation of the IAG and of CAG to DOPAC/DA ratio, found only in PE and with no such correlation in $\mathrm{E}$, further support the negative interference of the emotional and surgical stress-associated hypothalamic dopaminergic activation in the rapid IAG and CAG in PE following left Adx in DE-2.

Stress-associated dopaminergic activation in DE2 may be related to increased DA synthesis in the hypothalamus through an increase of 3,4-dihydroxyphenylalanine (DOPA) production, due to increased tyrosine hydroxylase $(\mathrm{TH})$ activity promoted by stressinduced rise in corticosterone ${ }^{30}$ and by inhibition of DA re-uptake by corticosterone. ${ }^{31}$ On the other hand, dopaminergic activity in E in left Adx, sham Adx and control rats was similar. This could be attributed, in part, to the suppression of TH mRNA levels by progesterone, as found in the arcuate nucleus in PE. ${ }^{32}$

Recent evidence suggests that estradiol $17 \beta$ may affect receptors in hypothalamic dopaminergic neurons by membrane initiated rapid signaling. ${ }^{33}$ Thus, a possible suppressive role of estrogens through a rapid effect on dopaminergic activity cannot be excluded. Estradiol $17 \beta$, by reducing dopaminergic activity in
PE through rapid signaling, may contribute to the significant CAG found in E. A positive correlation of the IAG to the uterine weight was found only in E. This finding is in line with a dependence of both IAG and uterine weight on a common factor, namely the preceding rise in estradiol levels.

Stress-associated dopaminergic activation in the hypothalamus in DE-2 might negatively interfere with CAG by affecting the efferent neural arc from the hypothalamus to the remaining adrenal. It is tempting to postulate that dopamine a-11 perikarya, located in the posterior hypothalamus sending axons to the spinal cord, ${ }^{34}$ were activated by the preceding stress. This might exert an inhibitory control in the context of homeostasis on the preganglionic fibres emerging from the thoracic segment of the spinal cord that activate the chromaffin cells in the medulla of the adrenal gland..$^{35}$

The attenuation of CAG and IAG by stress-associated dopaminergic activation after Adx in DE-2 may also be attributed to the activation of hypothalamic dopaminergic neurons of the arcuate and paraventricular nuclei that project into the intermediate lobe of the pituitary ${ }^{36,37}$ where DA receptors mRNA are highly expressed. ${ }^{34}$ This attenuation could be mediated in part through DA receptors inhibition of adenylyl cyclase, ${ }^{38}$ resulting in attenuation of cAMP accumulation and reduced stimulation of melanotrophs, the POMC producing cells in the intrermediate lobe of the pituitary. ${ }^{24}$ Thus, the stress-associated dopaminergic activation might result, in the context of homeostasis, in lower release of POMC derived $\gamma$-melanocyte stimulating hormone $(\gamma$-MSH) in DE-2. This decrease in $\gamma$-MSH attenuates its potentiating properties on the adrenal, namely hypertrophy and steroidogenesis. ${ }^{25,39}$ This notion is further supported by the present results suggesting that the enhancement of short-term CAG in E after left Adx in PE is partly mediated by estrogen and progesterone-driven reduction of dopaminergic activity. In favour of this assumption are previous ${ }^{32}$ and recent data showing that administration of estrogen to ovariectomized rats, followed by progesterone administration on the next day, decreases the dopaminergic activity in the intermediate lobe. ${ }^{40}$

We may conclude that our present results in 4- 
day cycling rats indicate a profound difference of short-term IAG and CAG between PE and E. It is suggested that the expected rise in $\mathrm{CRH}$, promoted by the rise of estradiol levels preceding left $\mathrm{Adx}$ in $\mathrm{PE}$, favours the within 19-20 h significant IAG and CAG observed in E. Moreover, the expected rise in progesterone in the afternoon of PE contributes to the enhancement of CAG and IAG by reducing stress-associated dopaminergic activation.

It is suggested that the hormonal background in DE-2 possibly promotes the chronic emotional plus surgical stress-associated hypothalamic dopaminergic activation and, together with the reduced release of N-POMC (1-74), the precursor of the mitogenic fragment in the adrenal, results in the profound attenuation of the CAG and IAG in PE.

Although results in animals may not have implications for humans, one may speculate that the present findings showing the role of estrogens in $\mathrm{CAG}$ and IAG might have certain relevance to humans undergoing unilateral adrenalectomy.

\section{REFERENCES}

1. Engeland WC, Ennen WB, Elayaperumal A, Durand DA, Levay-Young BK, 2005 Zone specific cell proliferation during compensatory adrenal growth in rats. Am J Physiol Endocrinol Metab 288: E298-E306.

2. Dallman MF, Engeland WC, Shinsako J, 1976 Compensatory adrenal growth: a neurally mediated reflex. Am J Physiol 231: 408-414.

3. Dallman MF, Engeland WC, Holzwarth MA, Scholz PM, 1980 Adrenocorticotropin inhibits compensatory adrenal growth after unilateral adrenalectomy. Endocrinology 107: 1397-1404.

4. Zarrow MX, Yochim JM, McCarthy JL 1964 The vaginal epithelium. In Experimental Endocrinology. Academic Press, New York and London; pp, 24-27.

5. Butcher RL, Collins WE, Fugo NW, 1974 Plasma concentration of LH, FSH, prolactin, progesterone and estradiol-17 $\beta$ throughout the 4-day estrous cycle of the rat. Endocrinology 94: 1704-1708.

6. Sfikakis A, Spyraki C, Sitaras N, Varonos D, 1978 Implication of the estrous cycle on conditioned avoidance behavior in the rat. Physiol Behav 21: 441-446.

7. Kazandjian A, Spyraki C, Sfikakis A, Varonos DD, 1987 Apomorphine-induced behaviour during the estrous cycle of the rat. Neuropharmacology 26: 1037-1045.

8. Sfikakis A, Galanopoulou P, Konstandi M, Tsakayannis D, 1996 Stress through handling for vaginal screening, serotonin and ACTH response to ether. Pharmacol
Biochem Behav 53: 965-970.

9. Sfikakis A, Papadopoulou-Daifotis Z, Sfikaki M, Messari J, 1998 Monoaminergic dysregulation on diestrus-2 and estrus through high emotional reactivity. Pharmacol Biochem Behav 60: 285-29.

10. Angeli A, Osella G, Ali A, Terzolo M, 1997 Adrenal incidentaloma: an overview of clinical and epidemiological data from the National Italian Study Group. Horm Res 47: 279-283.

11. Barzon L, Scaroni C, Sonino N, et al, 1999 Risk factors and long-term follow-up of adrenal incidentalomas. J Clin Endocrinol Metab 84: 520-526.

12. Kloos RT, Gross MD, Francis IR, Korobkin M, Shopiro B, 1995 Incidentally discovered adrenal masses. Endocr Rev 16: 460-484.

13. Kasperlik-Zeluska AA, Roslonowska E, Slowinska-Srzednicka J, et al, 1997 Incidentally discovered adrenal mass (incidentaloma): investigation and management of 208 patients. Clin Endocrinol (Oxf) 46: 29-37.

14. Sfikakis A, Papadopoulou-Daifotis Z, Bikas N, 2002 Inverse relationship of hippocampal serotonin to avoidance behavior, serotonergic activation by emotional stress differentiated by estrous cycle and surgical stress. Behav Brain Res 128: 41-52.

15. Bikas N, Gribisi J, Messari J, Sfikakis A, 2002 Compensatory adrenal growth in relation to stress of surgery and estradiol in adult male rats. Hormones (Athens) 1: 165-173.

16. Phillips R, Crock C, Funder J, 1985 Effects of mineralocorticoids and glucocorticoids on compensatory adrenal growth in rats. Am J Physiol 248: E450-E456.

17. Paxinos G, Watson C, 1998 The Rat brain in stereotaxic coordinates, Academic Press, California and London.

18. Sharp T, Zetterstrom T, Series HG, et al, 1987 HPLC-EC analysis of catechols and indoles in rat brain dialysates. Life Sci 41: 869-872.

19. Papadopoulou-Daifotis Z, Antoniou K, Vamvakides A, Kalliteraki I, Varonos DD, 1995 Neurochemical changes in dopamine and serotonin turnover rate in discrete regions of rat brain after the administration of glucinergic compounds. Acta Ther 21: 5-18.

20. McEwen BS, Alves SE, 1999 Estrogen actions in the central nervous system. Endocr Rev 20: 279-307.

21. Bohler Jr HCL, Zoeller RT, King JC, et al, 1990 Corticotropin releasing hormone mRNA is elevated on the afternoon of proestrus in the parvocellular paraventricular nuclei of the female rat. Brain Res-Mol Brain Res 8: 259-262.

22. Vamvakopoulos NC, Chrousos GP, 1994 Hormonal regulation of human corticotropin-releasing hormone gene expression: implications for the stress response and immune/inflammatory reaction. Endocrine Rev 15: 409420.

23. Buckingham JC, Dohler KD, Wilson CA, 1978 Activity of the pituitary-adrenocortical system and thyroid gland during the oestrous cycle of the rat. J Endocr 78: 359366. 
24. Lundblad JR, Roberts JL, 1988 Regulation of proopiomelanocortin gene expression in pituitary. Endocr Rev 9: 135-158.

25. Harmer SC, Bicknell AB, 2005 Role of gamma-MSH peptides in the regulation of adrenal steroidogenesis. Peptides 26: 1944-1951.

26. Lowry PJ, Silas L, McLean C, Linton EA, Estivariz FE, 1983 Pro- $\gamma$-melanocyte-stimulating hormone cleavage in adrenal gland undergoing compensatory growth. Nature 306: 70-73.

27. Estivariz FE, Iturriza F, McClean C, Hope J, Lowry PJ, 1982 Stimulation of adrenal mitogenesis by N-terminal pro-opiocortin peptide. Nature 297: 419-422.

28. Bicknell AB, Lomthaisong K, Woods RJ, et al, 2001 Characterization of a serine protease that cleaves pro- $\gamma$ melanotropin at the adrenal to stimulate growth. Cell 105: 903-912.

29. Bicknell AB, Lowry PJ, 2002 Adrenal growth is controlled by expression of specific pro-opiomelanocortin serine protease in the outer adrenal cortex. Endocr Res 28: 589595.

30. Dunn AJ, Gildersleeve B, Gray HE, 1978 Mouse brain tyrosine hydroxylase and glutamic acid decarboxylase following treatment with adrenocorticotrophic hormone, vasopressin or corticosterone. J Neurochem 31: 977982.

31. Gilad GM, Rabey JM, Gilad VH, 1987 Presynaptic effects of glucocorticoids dopaminergic and cholinergic synaptosomes. Implications for rapid endocrine-neural interactions in stress. Life Sci 40: 2401-2408.

32. Arbogast LA, Voogt JL, 1994 Progesterone suppresses tyrosine hydroxylase messenger ribonucleic acid levels in the arcuate nucleus on proestrus. Endocrinology 135: 343-350.

33. Malyala A, Kelly MJ, Ronneklew OK, 2005 Estrogen modulation of hypothalamic neurons: activation of multiple signalling pathways and gene expression changes. Steroids 70: 397-406.

34. Ben-Jonathan N, Hnasko R, 2001 Dopamine as a prolactin (PRL) inhibitor. Endocr Rev 22: 724-763.

35. Bowman WC, Rand MJ, 1982 The autonomic nervous system, neurochemical transmission and smooth muscle. In: Textbook of Pharmacology, $2^{\text {nd }}$ Edition, Blackwell Scientific publications, Oxford, London, Edinburgh, Melbourne; pp, 1-21.

36. Bjorklund A, Moore RY, Nobin A, Stenevi U, 1973 The organization of tubero-hypophyseal and reticulo-infundibular catecholamine neuron systems in the rat brain. Brain Res 51: 171-191.

37. Goudreau JL, Falls WM, Lookingland KJ, Moore KE, 1995 Periventricular-hypophyseal dopaminergic neurons innervate the intermediate but not the neural lobe of the rat pituitary gland. Neuroendocrinology 62: 147-154.

38. Missale C, Nash SR, Robinson SW, Jaber M, Caron MG, 1998 Dopamine receptors: from structure to function. Physiol Rev 78: 189- 225.

39. Al-Dujaili EA, Hope J, Estivariz FE, Lowry PJ, Edwards $\mathrm{CR}, 1981$ Circulating human pituitary pro- $\gamma$-melanocortin enhances the adrenal response to ACTH. Nature 291: 156-159.

40. DeMaria JE, Livingstone JD, Freeman ME, 2000 Ovarian steroids influence the activity of neuroendocrine dopaminergic neurons. Brain Res 879: 139-147. 